\title{
DESIGN AND CONSTRUCTION OF THE CONTROL SYSTEM FOR BATAN SMALL ANGLE NEUTRON SCATTERING SPECTROMETER (SMARTer)
}

\author{
E. Santoso and E.G.R. Putra \\ Center For Technology of Nuclear Industry Material \\ National Nuclear Egency Agency (BATAN) \\ Puspiptek Area, Serpong, Tangerang 15314 - Indonesia.
}

\begin{abstract}
DESIGN AND CONSTRUCTION OF THE CONTROL SYSTEM FOR BATAN SMALL ANGLE NEUTRON SCATTERING SPECTROMETER (SMARTer). A $36 \mathrm{~m}$ Small Angle Neutron Scattering (SANS) Spectrometer (SMARTer) has been installed in Serpong, Indonesia in 1992. As time goes by, the original main computer was out of order and the instrument had not been operated since 2003. In order to activate the SMARTer, in the year 2005, a work on designing and constructing a new control system for SMARTer was carried out. The main component of this control system is a programmable peripheral I/O (IC PPI 8255) and was assembled as a plug-in board at an ISA slot of a personal computer. An IC PPI 8255 was programmed to control the mechanical movements of the instrument's components: four neutron guide tubes, six pinholes collimator, a detector and a beam stopper. The test either with or without neutron beam has shown that this control system can be implemented for the mechanical movements of SMARTer. Error of moving the detector in the distance range of $1.5 \mathrm{~m}-18 \mathrm{~m}$ is only $1 \mathrm{~mm}$ and the other movements have no error at all (precise).
\end{abstract}

Keywords : Control system, Neutron spectrometer, Computer

\section{INTRODUCTION}

A small-angle neutron scattering (SANS) is widely applied for static and dynamic-structural analyses in materials science and biology fields such as in metallurgies, ceramics, polymers, colloids, vesicles, viruses, etc. in the spatial range of $1-100 \mathrm{~nm}$. It has been known that many important scientific findings were revealed by SANS technique for more than three decades[1]. Therefore, it could be understood that many SANS instruments are running all over the word and also in Serpong, Indonesia, that has a $36 \mathrm{~m}$ SANS spectrometer (SMARTer) which was commissioned in 1992[2].

The spectrometer has been completely installed at the end of a 49-meter long neutron guide and located in the neutron guide hall in neutron scattering laboratory (NSL). The SMARTer consists of $18 \mathrm{~m}$-long tube of collimator system which comprised of four sections of movable guide-tubes, one section of a fixed collimator (non-reflecting tube) and six circular pinholes collimator, Figure 1[3]. Meanwhile, another 18-meter long tube accommodates a $128 \times 128{ }^{3} \mathrm{He}$ two-dimensional position sensitive detector (2D-PSD) made by RIS $\emptyset$. The detector can be moved continuously from $1.5 \mathrm{~m}$ to $18 \mathrm{~m}$ from sample position and also shifted in lateral direction of 
$0.1 \mathrm{~m}$ to cover scattered beam at higher $Q$-range. Variation of collimator length and sample-to-detector distance (SDD) is fully controlled by computer. In 2003, the original main computer controlling of SMARTer was out of order, caused the SMARTer instrument had not been used for several years.

Provided with our experiences to build the new controller modules for several neutron instrument, i.e. four-circle diffractometer/texture diffractometer (FCD/TD), high-resolution powder diffractometer (HRPD) and triple-axis spectrometer (TAS)[4,5,6]. In 2005 the new SMARTer controller has been completely designed and constructed using local electronic components. The advantage of using local components are inexpensive, easy to get and be autonomous and not depending on other countries.

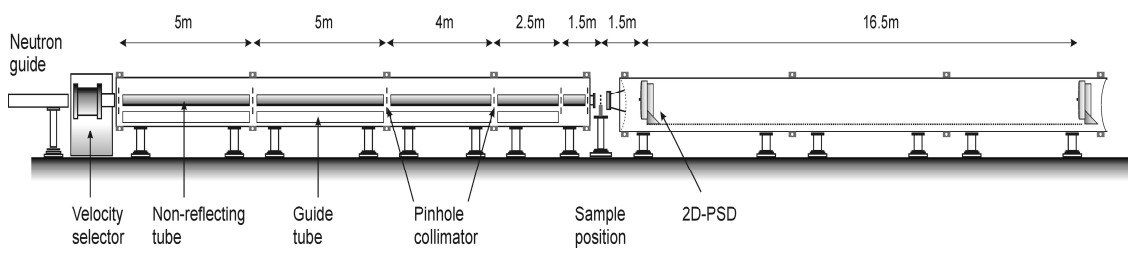

Figure 1. A schematic diagram of the $36 \mathrm{~m}$ SANS BATAN spectrometer (SMARTer) in Serpong.

\section{THEORY}

\section{SMARTer Controller}

To control the SMARTer instrument by the computer, an interface which has many programmable inputs/outputs (I/O) is needed. Due to SMARTer instrument using many stepper motors (18 motors), two pieces of IC programmable peripheral I/O (PPI 8255) have been used. The PPI 8255 was chosen as a main component because it is available in the local market, inexpensive and has a good stability.

The PPI 8255 has three ports called port A, port B and port C. Each of port $\mathrm{A}$ and $\mathrm{B}$ has eight pins and can be programmed as an input or output. Only port $\mathrm{C}$ has four bits Lower and four bits Upper. This each (four bits) can be programmed either as an input or output. For example, four bits as an input and four bits as an output or all eight bits either as an input or output. The selection of ports whether as the input or the output is chosen by the control register of the PPI 8255. This control register has eight bits which are divided into two groups; group B (bit 0-2), group A (bit 3-6) and 1 set flag (bit 7) as shown in Figure 2[7] 


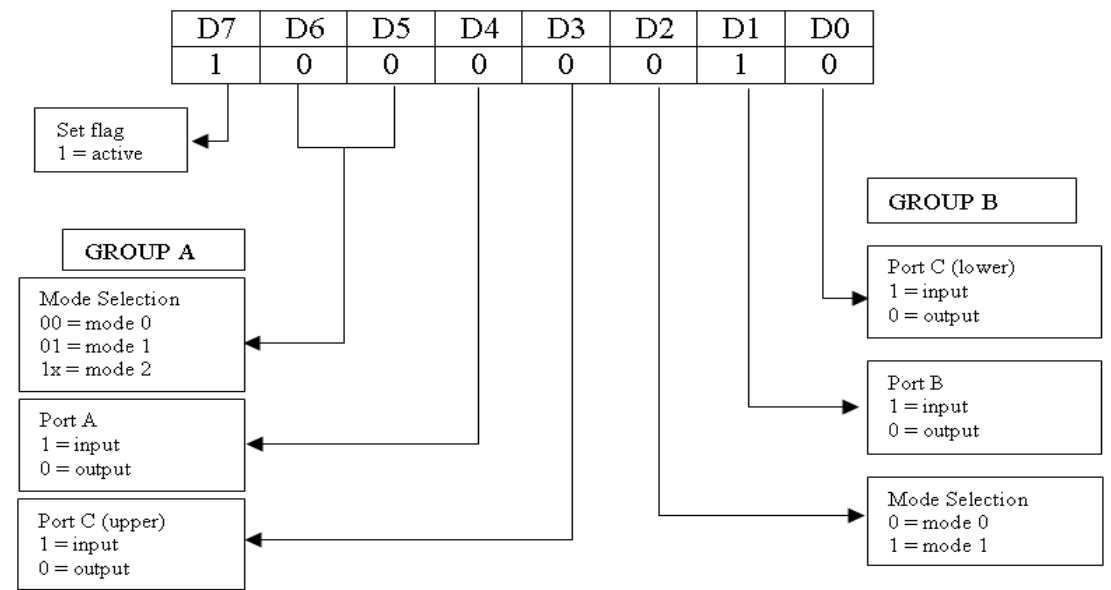

Figure 2. Procedure to fill the eight bits of control register PPI 8255.

\section{Block Diagram of SMARTer Control System}

The new SMARTer control system is more simple compare to the original ones made by Rigaku, Japan, as shown in Figure 3[8] and Figure 4. In Figure 4, it can be seen that the SMARTer control system consists of three groups of controller, i.e. collimator, pinhole and detector. Up and down movement of neutron guide tubes and non-reflecting tubes in collimation system, changing the pinhole apertures position, backward and forward movement of detector system are the main system in SMARTer that have to be working well for SANS experiments.

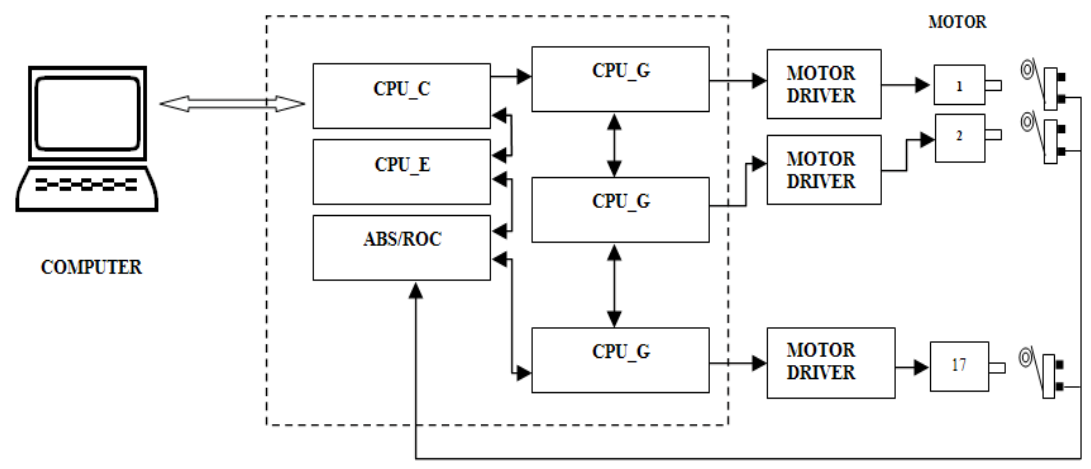

Figure 3. Block diagram of the original SMARTer control system. 


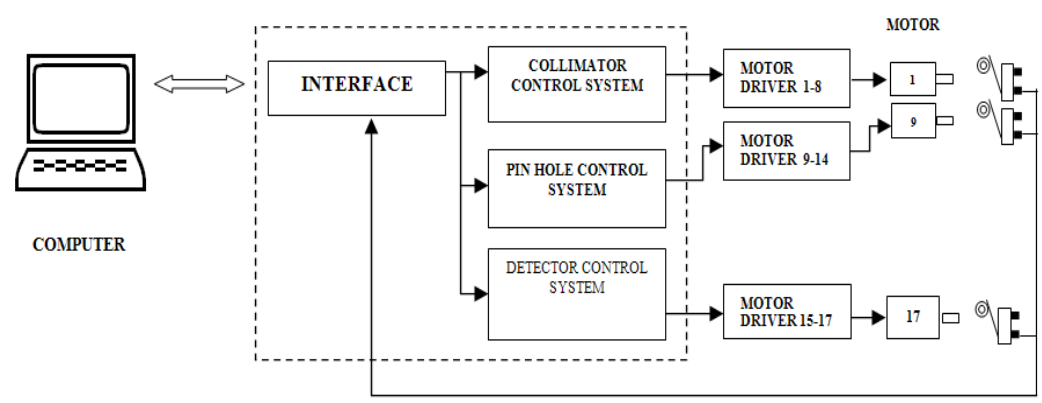

Figure 4. Block diagram of new SMARTer control system.

\section{RESULTS AND DISCUSSION}

After finishing the construction of SMARTer controller, each mechanical movement of SMARTer components was then tested by the special programed software. The testing result can be seen in Table 1,2 and 3 below.

From the testing results presented in the Table 1 and 2, it can be seen that no error is found because SMARTer was equipped with an absolute encoder on the detector positioning mechanism and optical sensor on the pinhole collimator.

It is clear that the position of the neutron guide tube for more than 10 times movements are same. It means that the mechanical movement of the neutron guide tube are very good and precise.

Table 1. Testing result of the position sensitive detector (PSD) movement.

\begin{tabular}{|c|c|c|c|c|}
\hline $\begin{array}{l}\text { Instruction/pr } \\
\text { ogram }\end{array}$ & $\begin{array}{c}\text { Detector Position } \\
{[\mathrm{mm}]}\end{array}$ & $\begin{array}{c}\text { Instruction/pr } \\
\text { ogram }\end{array}$ & $\begin{array}{c}\text { Detector Position } \\
{[\mathrm{mm}]}\end{array}$ \\
\hline Datum & 1500 & & $5 \mathrm{~m}$ & 5000 \\
\hline $2 \mathrm{~m}$ & 2000 & & $4 \mathrm{~m}$ & 4000 \\
\hline $3 \mathrm{~m}$ & 3000 & & $3 \mathrm{~m}$ & 3000 \\
\hline $4 \mathrm{~m}$ & 4000 & & $2 \mathrm{~m}$ & 2000 \\
\hline
\end{tabular}

Table 2. Testing result of a pinhole collimator alignment or centering.

\begin{tabular}{|c|c|c|c|}
\hline No. of Pinhole & Pin hole zise [mm] & Input (pulse or steps) & Cross position \\
\hline 0 & Closed & Datum & - \\
\hline 1 & 30 & Pinhole 1 & Good \\
\hline 2 & 20 & Pinhole 2 & Good \\
\hline 3 & 14 & Pinhole 3 & Good \\
\hline 4 & 10 & Pinhole 4 & Good \\
\hline 5 & 7 & Pinhole 5 & Good \\
\hline 6 & 5 & Pinhole 6 & Good \\
\hline 7 & $40 \times 90$ & Pinhole 7 & \\
\hline
\end{tabular}


Table 3. Testing result of up/down movement of a neutron guide tube.

\begin{tabular}{|c|c|c|c|c|}
\hline \multirow{2}{*}{$\begin{array}{c}\text { MOVEMENT } \\
\text { No. }\end{array}$} & \multicolumn{2}{|c|}{ DOWN } & \multicolumn{2}{c|}{ UP } \\
\cline { 2 - 5 } & PnOSITION & \multicolumn{2}{c|}{ POSITION } \\
\hline 0 & 80000 & 80000 & 79000 & 79008 \\
\hline 1 & 79901 & 79908 & 79100 & 79100 \\
\hline 2 & 79802 & 79808 & 79200 & 79200 \\
\hline 3 & 79702 & 79708 & 79300 & 79300 \\
\hline 4 & 79602 & 79608 & 79401 & 79400 \\
\hline 5 & 79502 & 79509 & 79500 & 79500 \\
\hline 6 & 79401 & 79408 & 79600 & 79600 \\
\hline 7 & 79301 & 79308 & 79700 & 79701 \\
\hline 8 & 79202 & 79208 & 79800 & 79800 \\
\hline 9 & 79101 & 79108 & 79900 & 79900 \\
\hline 10 & 79000 & 79008 & 80000 & 80000 \\
\hline
\end{tabular}

\section{Testing Using Neutron Beam}

The SMARTer instrument has been calibrated using several standard samples such as silver behenate (AgBE) for wavelength and momentum transfer $q$ calibration, porous silica (porasil CPG-10-75) and PS-PEP block copolymer samples for instrumental parameter and inter-laboratory comparison. The inter-laboratory comparison of SMARTer with SANS JAEA-Japan and ANSTO-Australia spectrometers on a silver behenate (AgBE) standard sample shows a good agreement on $q$ calibration, as shown in Figure 5[3]. The two-dimensional scattered pattern of $\mathrm{AgBE}$ is shown in Figure 6. The sample measurement data obtained from SMARTer have been subtracted with the background, noise and detector efficiency to provide

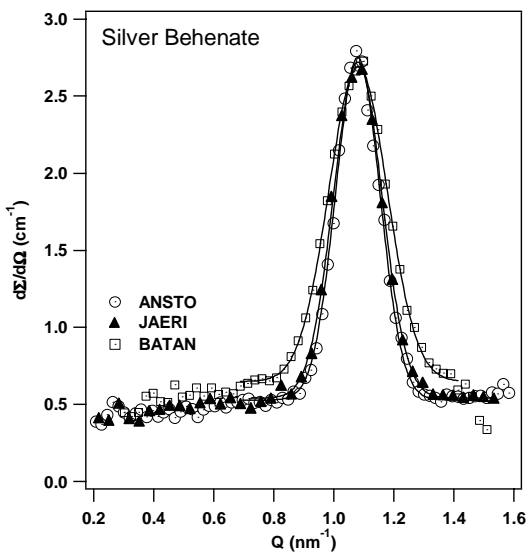

Figure 5. Silver behenate (AgBE) as a standard sample shows a sharp diffraction peak at $q=1.08 \mathrm{~nm}^{-1}$ or $0.108 \AA^{-1}$. 


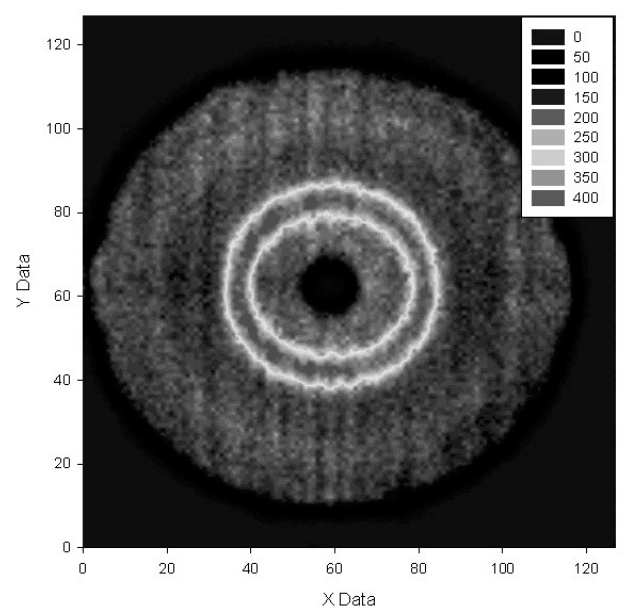

Figure 6. The two-dimensional scattered pattern of a first order of Bragg diffraction from silver behenate (AgBE) with sample to detector distance of $1.5 \mathrm{~m}(\lambda=3.90 \AA)$ for 18 hours exposed. Data was taken using SMARTer.

corrected scattering intensity in one or two-dimensional pattern. The data reduction program which is obtained from JAEA Japan under the bilateral cooperation between JAEA Japan and BATAN Indonesia was made originally by Jun-ichi Suzuki[9]. By now, the program has been modified and developed recently to suit the specification of SMARTer. Some experiments with several kinds of samples have been carried out and then compared to the other SANS spectrometer results in order to verify the performance of SMARTer.

Figure 7 shows that the performance of SMARTer in measuring micellar solutions sample is comparable to other SANS instruments at HANARO, KAERI, Korea.

The peak position $q_{\max }$ which is related to the inter-particle correlation factor appears at $0.085 \AA^{-1}$ for $0.3 \mathrm{M}$ SDS (Sodium Dodecyl Sulphate) micellar solutions. These results correspond to $74 \AA$ of average inter-particle distances between two micelles of SDS. The scattering data from BATAN had been analysed and fitted using data analysis program for micellar structure. Here, we obtained that the shape of the 0.3 M SDS is elongated with the ratio of minor and major axes about 1:1.65 and formed by $\sim 100$ of SDS molecules[10]. 


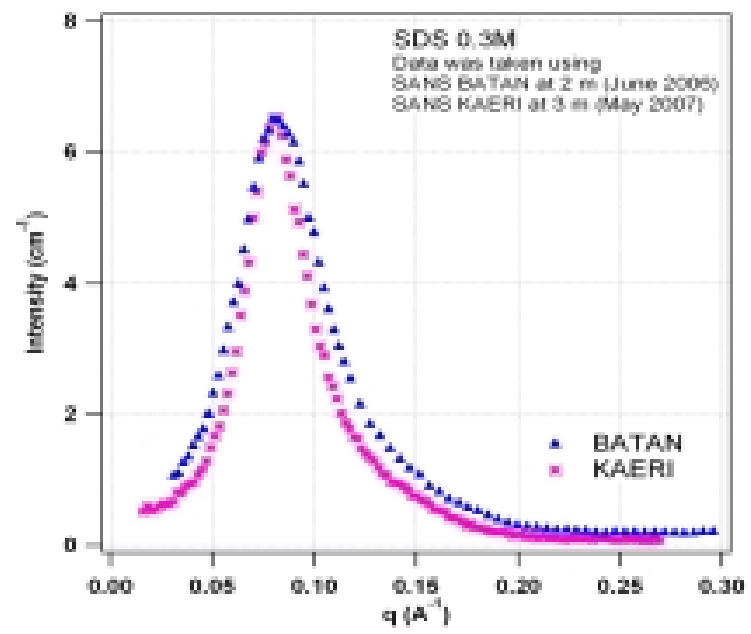

Figure 7. Comparison of SANS scattering profiles from 0.3M SDS solutions obtained by using SMARTer and HANARO SANS, KAERI, Korea.

The linearity of the detector was evaluated by carrying out SANS measurement of incoherent scattering from water of $1 \mathrm{~mm}$ thick at sample-to-detector distance of $1.5 \mathrm{~m}$ for 24 hours. For a linearity purpose, a cadmium plate with $5 \mathrm{~mm}$-circular pinholes was placed just in front of the detector and they are aligned on $100 \mathrm{~mm}$-pitch square arrays. The observed pinhole positions both in $\mathrm{X}$ and $\mathrm{Y}$ directions in pixel number are plotted against the actual pinhole positions. By measuring the distance among the transmitted neutron spots on the detector plane, a fairly good linearity of the detector was confirmed. The effective pixel size of $4.99 \mathrm{~mm}$ and $4.63 \mathrm{~mm}$ in $\mathrm{X}$ and $\mathrm{Y}$ directions respectively as reported elsewhere[11]. Meanwhile, the uniformity of the detector efficiency was examined using water of $1 \mathrm{~mm}$ thick in a quartz cell at sample-to-detector distance of $2.0 \mathrm{~m}$ for 10 hours. We employed a pixel-to-pixel correction of the sample data using data reduction software to subtract the scattering sample data with the backgrounds. The collected two-dimensional scattering intensity was corrected for the background scattering from quartz, and the electronic noise. The scaled radial averaging for detector efficiency showed that the detector efficiency is uniform over most of the detector area[11].

To cover the wide range of momentum transfer $q$, e.g. $0.01<q\left(\mathrm{~nm}^{-1}\right)$ $<1$, the detector must be placed at several sample-to-detector position. A ceramic sample was exposed in neutron beam with a wavelength of $3.9 \AA$ and then the scattered neutron was detected at several detector positions, Figure 8 . This measurement was carried out to perform the sensibility of the instrument in combining the data for several detector positions. Figure 8 shows that each scattering data taken at a fixed detector position matched 
precisely with other positions. Therefore, the wide $q$-range can be obtained that is very important to reveal the structure from the sample.

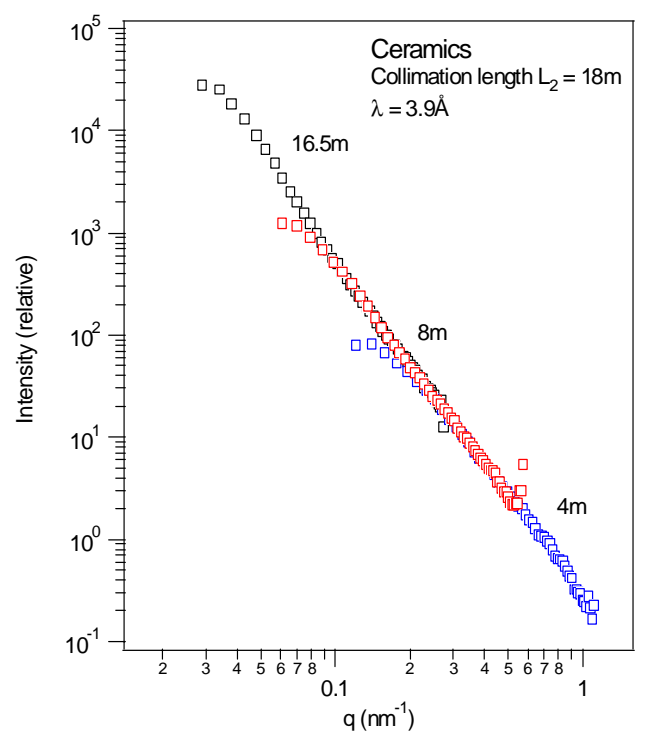

Figure 8. SANS distributions from a ceramic sample. Data was taken at several detector positions as indicated to cover a wide $q$-range. The result shows each data taken at fixed position matched with others.

Applying a fixed position beam stop is not fit properly in covering the deviated direct beam when the detector placed at the larger distance from the sample, $\mathrm{L}_{2}>4 \mathrm{~m}$, due to gravity, parasitic scattering or misalignment (centering problem). Using the large size of beam stop, such as $80 \mathrm{~mm}$ in diameter or more in front of the main detector will reduce the instrument capability to reach the lowest possible $q$ range. Here, a new two-mode motor controller on $\mathrm{X}$ and $\mathrm{Y}$ directions was employed to adjust a $30 \mathrm{~mm}$ thick boronated rubber beam stop with diameter $=60 \mathrm{~mm}$ in the center of direct beam. The beam stop adjustment can be controlled and remoted using the new control system of SMARTer. Detail results are reported elsewhere[11].

\section{CONCLUSION}

The testing result both with or without the neutron beams, showed that the SMARTer control system has the same result as that of the SANS instruments from ANSTO-Australia, JAEA-Japan and HANARO - KAERI, Korea. It was clear that a new SMARTer control system is working well to control the mechanical movement in SMARTer instrumental system and 
showing a good performance. By now, SMARTer is ready to use for experiments on nanostructure studies from a wide-range of samples in materials science and biology researches.

\section{ACKNOWLEDGEMENTS}

This work was supported by National Nuclear Energy Agency (BATAN) in 2005 financial year under the revitalization of BATAN's neutron scattering facilities project. The authors are delighted to Dr. A. Ikram, head of neutron scattering laboratory, PTBIN-BATAN for his helping and discussions on writing this paper.

\section{REFERENCES}

1. J.S. HIGGINS and H.C. BENOIT, "Polymers and Neutron Scattering", Clarendon Press, Oxford, (1994).

2. MARSONGKOHADI, Neutron News, 7 (2),12-16 (1996).

3. E. G. R. PUTRA, BHAROTO, E. SANTOSO, Y. A. MULYANA, Neutron News, 18 (1), 23-29 (2007).

4. E. SANTOSO, Construction of Alternative Control System for FCD/TD, Technical Report, P3IB - BATAN (2000).

5. E. SANTOSO and A. MAULANA, Construction of interfacing between High Resolution Powder Difraktometer (HRPD) and Personal Computer, Proceeding, The $5^{\text {th }}$ National Seminar on Neutron and X-Ray Scattering, 44-49, Serpong, Indonesia, 2 Juli (2003).

6. E. SANTOSO, Construction of Triple Axis Spectrometer (TAS) Controller, Technical Report, Balai Spektrometri, P3IB, BATAN, (2004).

7. A. WIDIATMO, H. EDUARD and FENDI, "Learning MicroprosessorMicrocontroler", by PT Elex Media Komputindo, Jakarta, Indonesia (1994).

8. ANONYMOUS, SANS (PM-105), Maintenance Manual (Hardware), Rigaku Co.,Tokyo, (1991).

9. J. SUZUKI, User Notes for the SANS-J, JRR - 3M, Part 1, JAERI, (1993).

10. E.G.R. PUTRA, A. IKRAM, Indonesian Juornal of Chemistry, 6 (2), 117-120 (2006).

11. E.G.R. PUTRA, BHAROTO, E. SANTOSO, A. IKRAM, "Improved Performances of $36 \mathrm{~m}$ Small-Angle Neutron Scattering Spectrometer BATAN in Serpong Indonesia" submitted to Journal of Nuclear Instruments and Method in Physics Research A, (2008). 